\title{
On the Influence of Emotion on Ovarian Reserve Function and Discussion on Diagnosis and Treatment
}

\author{
Na Zheng, Xiaoping Cui*, Rui Ma, Zuang Li, Xinchun Xiao \\ Shaanxi University of Chinese Medicine, Xianyang, Shaanxi, 712046, China.
}

\begin{abstract}
How to cite this paper: Na Zheng, Xiaoping Cui, Rui Ma, Zuang Li, Xinchun Xiao. (2021) On the Influence of Emotion on Ovarian Reserve Function and Discussion on Diagnosis and Treatment. International Journal of Clinical and Experimental Medicine Research, 5(1), 89-95.

DOI: 10.26855/ijcemr.2021.01.012
\end{abstract}

Received: December 31, 2020

Accepted: January 24, 2021

Published: February 4, 2021

*Corresponding author: Xiaoping Cui, Shaanxi University of Chinese Medicine, Xianyang, Shaanxi, 712046, China.

\begin{abstract}
With the rapid development of society, women have to bear multiple pressures from family, life and work, which virtually affect the function of ovarian reserve. In order to explore the relationship between emotional factors and ovarian reserve function, by analyzing the importance and harmfulness of emotional factors to ovarian reserve function, this paper highlights the need to soothe the liver and regulate qi in the whole process of treatment. The importance of emotional factors to ovarian reserve function is emphasized, and it is concluded that there is a significant clinical effect in the treatment of ovarian reserve function from the liver theory, and it is advocated that clinical attention should be paid to the regulating effect of emotion on ovarian reserve function. In order to achieve the purpose of harmony of qi and blood, at the same time, this paper is expected to provide a certain scientific basis for clinical treatment. Another case 1 is attached for corroboration.
\end{abstract}

\section{Keywords}

Ovarian Reserve Function, Emotion, Regulating Liver, Qi

Ovarian reserve function refers to the quantity and quality of eggs retained in the ovary, which is the key to ensure female fertility and reflects the reproductive endocrine function and reproductive potential of women [1]. Modern medicine believes that heredity, immunology, weight loss, contraceptive, environment, iatrogenic injury and other factors affect ovarian reserve function, but the specific pathogenesis is not clear, traditional Chinese medicine believes that kidney deficiency is the basic pathogenesis that affects the function of ovarian reserve. With the rapid social succession of high information, women have to bear the pressure of family, education of children, heavy economy, busy work and complex interpersonal communication, which virtually affect the function of ovarian reserve and seriously infringe upon women's physical and mental health and family happiness. At the same time, with the transformation of medicine to the biological-psychological-social model, the role of mental and psychological factors in the process of disease is becoming more and more prominent [2]. Treatment from the kidney alone has limitations, and emotional factors have been paid more and more attention by more and more experts and scholars.

\section{Emotion causes disease and serious injury to the liver}

"People have five Zang organs and five qi, and they are afraid of being happy, angry, worried and afraid". In the Song Dynasty, Chen Wuxuan's "prescription of extreme Diseases due to three causes” first added sadness and surprise to the five qi, that is, "seven emotions". The description of emotions in ancient books of traditional Chinese medicine involves five emotions and seven emotions: anger injures the liver, surprise, joy and sadness, thinking of 
injuring the spleen, sorrow and sorrow injuring the lungs, fear of injuring the kidney, which is closely related to the five internal organs. Wang Mengying said in the Qing Dynasty: "the disease of seven emotions must start from the liver". Pointing out that the emotion is not smooth, it is most likely to lead to stagnation of liver qi, obstruction of qi and blood and disease. Related studies have also proved that the liver accounts for the highest proportion of menstrual diseases induced by viscera dysfunction, so emotion as the primary influencing factor should be paid attention to [3].

\section{On the influence of emotion on Ovarian Reserve function}

\subsection{Understanding of traditional Chinese medicine}

Emotion is a special term for traditional Chinese medicine, which is not only the reflection of the human body to external stimuli on the spiritual level, but also one of the external manifestations of the functional activities of Zang-fu organs [4]. "Female secretaries want to roll up the four original meridians", Yun said: "most women are stubborn, remote, angry and jealous, and hurt the liver". Thus, it can be seen that emotion causes disease, liver depression and qi stagnation, which is related to the characteristics of women who are kind-hearted and depressed. "Lingshu five-tone and five-flavor" cloud "the birth of a woman, more than qi, less than blood, removing blood by its number”, coupled with the special physiology of menstruation, belt, fetus, birth, milk and so on, which greatly increases the morbidity of emotion [5].

\subsubsection{Women take Qi as the communication-more than Qi}

Ye Tianshi proposed that "women are born with the liver". The liver qi is regulated, the meridians are smooth, and the sea of blood is quiet. However, because women are good and depressed, often have more than qi, the liver qi is easy to stagnate, and then hurt in the blood, Qi points are more prosperous, so that the liver is out of tune, catharsis is excessive, Chong Ren is out of charge, and the sea of blood is overflowing with irregular menstruation; Qi depression for a long time, irregular menstruation, dysmenorrhea, gradually amenorrhea, infertility; Chong Ren loss of smooth, postpartum qi stagnation milk is lack of milk. As "all diseases are inseparable from depression, all depression belongs to the liver".

\subsubsection{Women use blood-Less than blood}

Women use blood and are born with pure yin, and the special physiology of uterine meridian, belt, fetus, birth, milk and so on all depend on the nourishment of blood, so the blood is often insufficient, then the liver blood is easy to be deficient. Traditional Chinese medicine believes that "Qi is the handsome of blood" and "blood is the mother of Qi”. Qi and blood coordinate and use each other. If the woman's feelings are not comfortable, the liver qi is stagnant, which affects the upper and lower qi of the whole body, resulting in poor operation of qi and blood, qi stagnation and lack of essence and blood in the uterus, then the amount of menstruation is less, later stage, amenorrhea, infertility; if the qi and blood is not filled to support the fetus, then abortion, miscarriage; postpartum blood loss, Chong Ren deficiency of qi and blood, no way to turn breast-feeding. In addition, the liver and kidney are the viscera of the mother and son, and the liver is the kidney, which is homologous to the essence and blood, while the deficiency of the liver and blood leads to the deficiency of the kidney, which affects the kidney, destroys the balance of the kidney-Tiangui-Chong Ren-uterus, and affects the function of the ovary [6].

\subsection{Knowledge of western medicine}

On the basis of previous generations of physicians, modern medicine also has related exploration and research. Epidemiological investigation shows that the patients with decreased ovarian reserve function show the characteristics of high education and mental work [7]. Due to long-term work pressure, patients show psychological changes such as anxiety and depression, interpersonal sensitivity, environmental maladaptation and so on, which interfere with hypothalamus-pituitary-ovary axis (HPO axis) and affect female reproductive endocrine function. Modern medicine believes that emotion is the result of the joint action of the cerebral cortex and the limbic system, in which the hypothalamus is directly involved in the emotional behavior response. Long-term negative emotional stimulation can not only break the balance of pituitary-ovary axis by affecting the level of hypothalamus, but also directly affect ovarian function [8]. When women encounter psychological, social and other physiological stress, corticotropin-releasing hormone (CRH) secreted by hypothalamus inhibits gonadotropin-releasing hormone (GnRH) pulse release rhythm [9-10], which reduces the level of gonadotropin, which can lead to amenorrhea and infertility [11].

Western medicine believes that the liver is detoxified in a sleeping state from 11:00 pm to 1:00 am, and modern women are under overload pressure. Staying up late at night has become the norm, affecting liver detoxification and 
interfering with the biological clock and endocrine level in the body. Hong Jun, Lin Huaner et al. [12-13] investigation shows that biological clock disorder can lead to emotional deterioration, directly affect the level of hormones and reduce immunity, and induce a variety of gynecological diseases Cymbal.

\section{On the diagnosis and treatment of Ovarian Reserve function from the Perspective of emo- tion}

With the mature development of medical bio-psycho-social model, psychological and emotional factors play a more and more important role in gynecological diseases. Professor Zhang Boli [14] found that the law of the spread of emotional diseases is as follows: emotion causes disease, the first disease is in the liver, the disease spreads in the heart, and it gradually spreads to its internal organs, resulting in the loss of liver and blood storage, discord among the five internal organs, and a variety of gynaecological and obstetrical diseases. Therefore, women's diseases are mostly based on the liver theory, smooth and easy emotion, reconcile qi and blood, acupuncture and medicine, adapt to the cycle, and adjust the biological clock in order to improve the ovarian reserve function.

\subsection{Regulating emotion, psychotherapy}

Traditional Chinese medicine believes that heart disease still needs cardiac medicine, in which "heart disease" is different from the heart disease of modern medicine, which refers to the morbid state of bad mood depression and nowhere to vent for a long time. Unlike traditional diagnosis, doctors are in the process of treatment. To play the role of a listener, it is necessary to establish good psychological communication with patients, know what they are confused, help them vent their depression, release bad emotions, make them correctly understand the disease, treat the disease and receive treatment with a positive attitude, and at the same time popularize the importance of emotion to the disease to the family members, so that the family can fully understand and understand the patient's psychological state and clinical manifestations, and be considerate, caring and tolerant to the greatest extent. Actively guide them out of the haze of mind, which is also in line with the "emotion-winning therapy" of traditional Chinese medicine (such as pleasing emotion, empathy, victory over emotion, passion), that is, using one emotion to restrain another emotion, in order to reduce and eliminate bad emotions. Some scholars in the treatment of patients with menstrual disorders caused by liver depression, on the basis of Yangyin Shugan capsule, guide them to watch comedies and empathy after 3 months, the total effective rate is 92.9\% [15]. It can be seen that emotion is not only a pathogenic factor, but also an important means of treatment, mutual cause and effect, we need to seek advantages and avoid disadvantages, in order to get twice the result with half the effort.

\subsection{Traditional Chinese medicine treatment}

Many experts and scholars have also realized the importance of emotion in women's diseases, mostly from the liver theory, the curative effect is remarkable. Ban Xiuwen, a master of traditional Chinese medicine, believes that no matter what kind of emotion, it often causes more depression among women, and the regulation and treatment of gynecological diseases take soothing the liver and regulating qi as the great method, and often choose Chaihu Shugan San, Xiaoyao Powder and so on [16]. Yang Guiyun and du Baojun made good use of Bupleurum and other drugs to regulate the liver and soften the liver to improve ovarian reserve function [17-18]. Yuan Xieying [19] by comparing the normal ovarian reserve group, the decreased group and the premature senility group, it was found that the proportion of irritability and depression in the latter two groups was significantly higher than that in the normal group. Cai Yumei, Miao Xiaoling et al. [20], 60 patients with decreased ovarian reserve function were randomly divided into treatment group $(n=30)$ and control group $(n=30)$. The control group was treated with estradiol valerate tablets/estradiol cyproprogesterone tablets. After 3 months, it was found that the total effective rate of the treatment group was significantly higher than that of the control group, and the traditional Chinese medicine for tonifying the kidney and soothing the liver could regulate menstruation and significantly improve the reduced ovarian volume.

\subsection{Clockwise, attach importance to regulating the liver}

In the later stage of menstruation: nourishing blood and softening liver, supplemented by products that replenish qi. At this time, after menstruation, the uterus is empty and yin blood is insufficient, so we should pay attention to nourishing blood and softening the liver, regulating qi and promoting the regeneration of essence and blood of uterine uterus. Siwu decoction combined with Xiaoyao Powder (15g of cooked land, $12 \mathrm{~g}$ of Angelica sinensis, $10 \mathrm{~g}$ of Chuanxiong, 10g of Radix Paeoniae Alba, 10g of Bupleurum, 15g of Atractylodes macrocephala, 10g of Poria co- 
cos, 15g of yam, 3G of Ejiao, 10g of sour jujube seed), supplemented with Radix Astragali, Codonopsis pilosula, Zihe car and other replenishing qi, nourishing blood and regulating menstruation, to help. In addition, due to the low immunity of post-menstrual patients, inflammation is easy to occur, at this time should be heat-clearing, dehumidification and detoxification drugs, preventive treatment, improve the internal environment to regulate menstruation and assist pregnancy; a small amount of Qi-tonifying drugs to strengthen the positive and remove evil, improve immunity [21].

Premenstrual period: soothing the liver and regulating qi, supplemented by warming and invigorating blood circulation. At this time, due to the approaching menstruation, the rapid withdrawal of estrogen and progesterone may affect women's mental psychology, so it is necessary to soothe the liver and regulate qi, avoid the effect of emotion on the hypothalamic reproductive axis, reduce the function of ovarian reserve, cause menstrual disorder or induce ovulation disturbance. Add or subtract Shugan Jieyu decoction (Bupleurum 10g, Xiangfu 12g, tulip 10g, Qingpi $10 \mathrm{~g}$, Salvia miltiorrhiza $10 \mathrm{~g}$, Chuanxiong $10 \mathrm{~g}$, Corydalis $10 \mathrm{~g}$, toosendan $10 \mathrm{~g}$ ). At the same time, add a little fennel, Achyranthes bidentata, chicken blood vine, Angelica, motherwort and other temperature to relieve pain. This may be related to the traditional Chinese medicine for soothing the liver and tonifying the kidney to improve endometrial blood flow and uterine artery blood perfusion, adjust the local blood supply of the ovary, regulate the microenvironment and improve the ovarian function.

\subsection{Acupuncture treatment}

The palace is the "Qi Heng fu-fu". The three pulse of Ren Chong-du starts from the cell palace, also known as "one source and three differences", which plays a remote role in regulating the cell palace. Liver meridian "around the yin, to the lower abdomen", and intersects with the three pulse, clinical moxibustion liver menstruation and other gynecological diseases. Chen Wei's team [22] of acupuncture and moxibustion for tonifying the kidney and regulating the liver were used to treat patients with decreased ovarian function, and the curative effect was not significantly different from that of the western medicine group, and avoided hormone replacement therapy to increase the risk of breast cancer, endometrial cancer, cervical cancer and thrombotic diseases. This may be due to the estrogenic effect of acupuncture, which can increase $E_{2}$ levels and reduce FSH, thus improving ovarian reserve function and relieving clinical symptoms. According to kidney essence deficiency, Chong Ren imbalance and poor emotion, Shang Jie and Fang Jingong selected Baihui, Shenting, Benshen, Zhongwan, Tianshu, Guanyuan, Dahe, ovary, Zusanli, Sanyinjiao and other acupoints to treat DOR, including Baihui, Shenting, Benshen, Taichong soothing the liver and regulating mind, at the same time, instructing regular work and rest, regulating emotion and light diet, and the patient's sex hormone level was normal after 3 cycles. Depressive symptoms significantly improved ovarian reserve function [23].

\subsection{Adjust the biological clock, the unity of man and nature}

Traditional Chinese medicine attaches importance to the overall concept and pays attention to the unity of man and nature, just as the book "the formation of the five Zang organs" says, "if you lie, the blood belongs to the liver". When you defend Qi (23:00 to 1), it runs in Yin, which coincides with Ying Qi, so that more blood flows to the liver, and the essence and blood of the liver is harmonious. The mood is free and easy. If the child does not sleep, or insomnia, can cause liver loss and catharsis, Yin blood cannot return to the liver, irritability, headache, dizziness, eye astringency, tinnitus, or depression, paranoia and other emotional disorders [24]. This is consistent with the disorder of biological clock in modern medicine, which leads to the disorder of human corticosteroids and sex hormones [25-26]. Therefore, women should unite heaven and man, adjust the biological clock, balance yin and yang, avoid staying up late, consume liver and blood. Only then can Chong Ren be reconciled and there is blood in the uterus in order to improve the function of the ovary and become pregnant.

\section{Typical medical record}

Wang, female, 32 years old, doctor, married. First visit on April 27, 2020.

The patient complained that the menstruation was reduced by 5 years and the cycle was delayed by 23 days. Self-report 13-year-old menarche, past menstruation, since 2015, the number of menstruation gradually decreased, cycle 28-30 days, menstruation 3 days, a small amount (each time a total of 3-4 pieces of sanitary napkins), a small amount of blood clots, accompanied by premenstrual breast pain, a small amount of hair loss, untreated. A year and a half ago cesarean section 1 female, postpartum depression, after treatment is now improved, often stay up late overtime. I had insomnia after quarreling with my family a month ago, my menstruation was postponed for a week, and I took progesterone capsule for 5 days before menstruation. Now I came to the affiliated Hospital of Shaanxi 
University of traditional Chinese Medicine to seek TCM recuperation. LMP: 2020-3-3, this is the 56th day of the menstrual cycle, hair loss, face, poor spirit, poor appetite, poor night rest, two stools. The tongue is red, the fur is yellowish, pointed red, and the pulse strings are numbered. B-mode ultrasonography showed that the size of uterus was about $7.6 \mathrm{cmx} 5.5 \mathrm{~cm} \times 3.1 \mathrm{~cm}$, the size of right ovary was about $3.2 \mathrm{~cm} \times 2.7 \mathrm{~cm}$, the size of right ovary was about $3.2 \mathrm{~cm} \times 2.7 \mathrm{~cm}$, the size of left ovary was about $3.3 \mathrm{~cm} \times 1.9 \mathrm{~cm}$, and there were 7 dark areas of $0.5 \mathrm{~cm}$ follicles, which were about $1.3 \times 1.1 \mathrm{~cm}$. Six sex hormones: FSH: $3.8 \mathrm{mIU} / \mathrm{mL}, \mathrm{LH}: 4.6 \mathrm{mIU} / \mathrm{mL}, \mathrm{E2}: 44 \mathrm{pmol} / \mathrm{L}$, P: 2.2nmol/L, T:1.21nmol/L, PRL:0.27nmol/L. Blood $\beta$-HCG $<0.100 \mathrm{mIU} / \mathrm{mL}$. Western medicine diagnosed as menstrual disorders. The diagnosis of traditional Chinese medicine is: 1. The amount of menstruation is less than; 2. Late menstruation; 3. Hair loss. Dialectical: liver depression and blood deficiency. Treatment: soothing the liver and relieving depression, reconciling qi and blood. Prescription: Siwu Xiaoyao Powder combined with tonifying female and promoting endometrium is added and subtracted. The specific medication is as follows: (1) Radix Bupleuri 12g, Chuanxiong 10g, Radix Paeoniae Alba 10g, Atractylodes macrocephala 15g, Angelica 12g, turmeric 10g, Xiangfu 15g, toosendan 10g, astragalus 30g, Codonopsis pilosula 15g, yam 15g, Fructus Aurantii 10g, ginseng 10g, dodder 15g, Dipsacus 15g, Fushen 10g, Yuanzhi 20g, Yuanzhi 15g, Jiao Hawthorn 10g, Jiao Shenqu 10g, Jiao malt 10g. At the same time, Jiao Sanxian is used to strengthen the spleen and appetizer, promote digestion, Poria, Yuanzhi, night rattan to improve sleep and other symptoms. (2) to guide patients to appeal, tell them to feel free, strengthen nutrition and avoid staying up late. (3) Xiaoyao pills, orally, 8 pills per time, three times a day. (4) B-ultrasound was reexamined next week to monitor the growth of endometrium.

On May 3, the second visit: LMP: 2020-3-3, this is the 62nd day of the menstrual cycle. B-ultrasound showed that the size of the uterus was about $8.0 \mathrm{~cm} \times 5.3 \mathrm{~cm} \times 2.9 \mathrm{~cm}$, the thickness of the endometrium was slightly hyperechoic, the size of the right ovary was about $3.1 \mathrm{~cm} \times 2.9 \mathrm{~cm}$, there were 5 dark areas of $0.5 \mathrm{~cm}$ follicles, and the size of the left ovary was about $3.0 \mathrm{~cm} \times 2.1 \mathrm{~cm}$, in which 4 dark areas of $0.5 \mathrm{~cm}$ follicles could be seen. After taking traditional Chinese medicine, the patient's diet was significantly improved, his mental state improved, it was still difficult to fall asleep, there was no other discomfort, the tongue was red, the fur was thin and white, and the pulse was slippery. The prescriptions are as follows: add $20 \mathrm{~g}$ of wild jujube kernel on the basis of the front, increase the calming power to improve sleep, a total of 3 doses, 1 dose per day, warm in the morning and evening.

May 9: LMP: 2020-5-4, this is the 5th day of the menstrual cycle, self-reported oral Chinese medicine menstruation on the 2nd day, 3 days net, less, dark color, more blood clots, accompanied by premenstrual breast pain. Tell it (1) to add and subtract Siwu Xiaoyao San and Zuogui pills (Bupleurum 12g, Chuanxiong 10g, Radix Paeoniae Alba 10g, Atractylodes macrocephala 15g, Angelica sinensis 12g, turmeric 10g, Xiangfu 15g, toosendan 10g, Huangqi 30g, Codonopsis pilosula 15g, yam 15g, Fructus Aurantii 10g, ginseng 10g (other package), dodder 15g, Dipsacus 15g, Chinese wolfberry 10g, Gentiana 10g, dandelion 10g, Zhigan. (2) Meridian Shugan Jieyu decoction combined with Yougui pills (Bupleurum 10g, Xiangfu 10g, Yujin 10g, Qingpi 10g, Chuanxiong 10g, Salvia miltiorrhiza 10g, Zelan 10g, toosendan 10g, Corydalis 10g, cooked aconite 3G (baked service), cinnamon 9g, Cornus meat 10g, yam 15g, dodder 10g, Eucommia ulmoides 10g, Angelica 10g, Achyranthes bidentata 10g, chicken blood vine 10g, motherwort. Instruct patients to recuperate 2 menstrual cycles according to this plan, and at the same time have a smooth mood, work and rest regularly, and cultivate interests and hobbies to decompress.

The fourth clinic on July 12: LMP: 2020-7-8, this is the 5th day of menstrual cycle, 28-30 days of menstrual cycle, menstruation volume is significantly increased than before treatment, 4 days menstrual net (a total of 10 sanitary napkins), the patient's spirit is good, look cheerful, add a little new hair, take food, improve night rest, and adjust second defecation.

Note: the patient has doctoral degree, has symptoms of significantly reduced menstruation and prolonged cycle, has a history of postpartum depression, hair loss and bad habits such as often staying up late in the past year, and then auxiliary examination shows that the patient's ovarian function has declined, probably because the patient is a highly knowledgeable person, engaged in more mental work, often stay up late, postpartum blood injury, consumption of fine blood, resulting in insufficient blood supply to the hypothalamus, affecting ovarian function through the HPO axis. The occurrence of hair loss, less menstruation, coupled with the quarrel with the family half a month ago led to its liver qi stagnation, qi stagnation and blood stasis, so late menstruation, less amount, dysmenorrhea. According to the pathogenesis of liver depression and blood deficiency and conforming to the menstrual cycle, the author gives Siwu Xiaoyao Powder in the later stage of menstruation to nourish yin and nourish blood, soothe the liver and regulate qi to promote the regeneration of new blood and the development of follicles, soothe the liver and warm the kidney, activate blood circulation and dredge menstruation in the early stage of menstruation to help the excretion of follicles or intima. The whole process paid attention to soothing the liver and regulating qi, while improving ovarian function and concomitant symptoms. After the regulation and treatment of 2 menstrual cycles, the 
patient's menstruation was regular, the amount of menstruation was significantly increased, and the symptoms were alleviated.

\section{Summary}

With the development of high information technology in society, emotional factors play a more and more important role in female diseases, which can be used as both pathogenic factors and treatment methods. Bad mood has negative feedback regulation on female ovarian reserve function, which can cause menstrual disorder, amenorrhea, infertility and so on. Traditional Chinese medicine believes that emotion causes disease, which mostly involves the liver, the catharsis and adjustment of the liver, the harmony of qi and blood, and ease of spirit. in clinic, attention should be paid to the influence of emotion on the reserve function of ovary, soothing the liver and regulating qi in the whole process, and regulating qi, as the saying goes, "Qi is regulated by itself”. After adjustment, the blood is often foot. Western medicine believes that we should pay attention to psychological communication with patients, adopt comfort, sympathy, enlightening words, and induce them to release bad emotions, increase mutual trust, and promote the establishment of a new type of doctor-patient relationship. However, the mechanism of TCM treatment from emotion theory on ovarian reserve function is not clear, and there is a lack of experimental research support, only this article is expected to provide new ideas for diagnosis and treatment in the future.

\section{References}

[1] Han Jingpei, Summer. (2019). Research progress on the pathogenesis of decreased ovarian reserve function in traditional Chinese and western medicine [J]. Hunan Journal of traditional Chinese Medicine, 35(03): 157-160.

[2] Jiang Huifang. (2008). Clinical significance of treating body and mind as a whole in traditional Chinese Medicine [C]/the sixth Annual meeting of the Health Care and Rehabilitation Medicine Committee of Zhejiang Society of Integrated traditional Chinese and Western Medicine and the compilation of national continuing education classes.

[3] Diao Juncheng. (2008). Study on the basic theory and treatment methods of syndrome differentiation in the treatment of menstrual disease from the liver [D]. Changsha: Hunan University of traditional Chinese Medicine, 2008.

[4] Yang Shaohui. (2012). Study on the Origin of emotional Pathogenesis and the relationship between emotional factors and Gynecological Diseases [D]. Thesis of Guangzhou University of traditional Chinese Medicine, 2012: 3mur10.

[5] Cui Xiaoping. (1995). On the connotation of "Women are born with the liver" [J]. Journal of Shaanxi College of traditional Chinese Medicine, 1995(01): 8-10.

[6] Ge Lingling, Hu Lan Yawen. (2019). Research progress on the relationship between emotional disorder and the decline of ovarian reserve function [J]. New traditional Chinese Medicine, 2019, 51(04): 46-49.

[7] Xu Cai. (2011). Study on the cause, mechanism, syndrome and treatment of the decline of ovarian reserve function based on social-logical factors [D]. Reform and Opening Up, 2011, Jing 3(8): 134-136.

[8] Luo Laicheng, Huang Rongbo, Wang Jianhong. (2009). Artificial neural network was used to study the disorder mechanism of hypothalamus-pituitary-ovary axis in patients with abnormal emotional activity [J]. Shi Zhenguo Medicine, 2009 Journal, 20(5): 1064-1066.

[9] Frattarelli, J. L. B. P. A. D. (2000). Evaluation of basal estradiol levels in assisted reproductive technology cycles [J]. Fertility and Sterility, 2000, 74(3): 518-524.

[10] Mann E., Singer D., Pitkin J., et al. (2012). Psychosocial adjustment in women with premature menopause: a cross-sectional survey [J]. Climacteric: The Journal of the International Menopause Society, 2012, 15(5): 481-489.

[11] Marinelli, P. W., Quirion, R., Gianoulakis, C. (2004). An in vivo profile of beta-endorphin release in the arcuate nucleus and nucleus accumbens following exposure to stress or alcohol [J]. Neuroscience, 2004, 127(3): 777.

[12] Hong Jun, Ma Wenlong. (2001). Effects of total sleep deprivation on mood of healthy young men [J]. Chinese Journal of Psychiatry, 2001, 34(3): 176-179.

[13] Lin Huan-er, Chen Ai-ying, Fu Peifang, et al. (2006). study on the relationship between psychosocial factors and female post-puberty acne [J]. International Medical and Health Bulletin, 2006, 12(7): 4-5.

[14] Zhang Liping, Zhang Boli. (2008). Analysis and reflection on the present situation of traditional Chinese Medicine Research on emotional Diseases [J]. Liaoning Journal of traditional Chinese Medicine, 2008 Journal of Chinese Medicine, 35(3): 349.

[15] Cao Junhong. (2016). Clinical effect of TCM psychosomatic therapy on menstrual disorders in women with decreased ovarian reserve function [J]. Continuing Medical Education in China, 2016, 8(8): 180-181.

[16] Liu Yuyun, Dai Ming, Dong Lan, et al. (2013). Professor Ban Xiuwen’s experience in treating gynecological diseases from emotion [J]. Journal of traditional Chinese Medicine, 2013, 28(10): 1476-1478.

[17] Li Zhen Li. (2015). Yang Guiyun’s experience in treating gynecological diseases from the liver [J]. Jiangsu traditional Chinese 
Medicine, 2015, 47(7): 29-30.

[18] Cao Cuiling. (2016). Professor du Baojun's experience in treating the decline of ovarian reserve function [D]. Beijing: Beijing University of traditional Chinese Medicine, 2016.

[19] Yuan Xieying. (2016). Clinical related factors affecting premature ovarian failure and decreased ovarian reserve function [J]. Chinese and Foreign Women’s Health Study, 2016, (8): 209214.

[20] Cai Yumei. (2017). Clinical observation of the method of nourishing kidney and soothing liver in the treatment of decreased ovarian reserve function [D]. Yunnan College of Traditional Chinese Medicine, 2017.

[21] Zou Wenjun, Li Jiyan, Li Zhaohui, Ma Yao, Xie Jianguo. (2019). Discussion on the treatment of dysovulatory infertility from Qi Theory [J]. Global traditional Chinese Medicine, 2019, 12(08): 1245-1247.

[22] Chen Wei. (2018). Clinical observation of acupuncture and moxibustion tonifying kidney and regulating liver on decreased ovarian reserve function of kidney deficiency and liver depression [D]. Hunan University of traditional Chinese Medicine, 2018.

[23] Shang Jie, Fang Xinggong, Li Xiaotong, et al. (2018). A brief analysis of "flirting and good luck" in the treatment of decreased ovarian reserve function [J]. Global Chinese Medicine, 2018, 11(6): 876-879.

[24] Yang Bilian. (2014). Discussion and syndrome differentiation treatment of adult female acne caused by biological clock disorder [J]. Global traditional Chinese Medicine, 2014, 7(01): 37-Qing.

[25] Wang Shengxu, Li Qiushi. (2002). Effects of sleep deprivation on the content of amino acid neurotransmitters in rat brain [J]. Journal of the First Military Medical University, 2002, 22(10): 888-890.

[26] Sun Qingmei, Li Dongyan, Feng Tiejuan, et al. (2009). Effects of sleep deprivation on sex hormone levels in puberty rats [J]. Chinese School Medical Officer, 2009, 23(3): 269-270.

[27] Hong Jun, Ma Wenlong. (2001). Effects of total sleep deprivation on mood of healthy young men [J]. Chinese Journal of Psychiatry, 2001, 34(3): 176-179. 